\title{
Optimal Gaussian density estimates for a class of stochastic equations with additive noise
}

\author{
David Nualart * \\ Department of Mathematics \\ University of Kansas \\ Lawrence, Kansas, 66045, USA \\ nualart@math.ku.edu
}

\author{
Lluís Quer-Sardanyons $^{\dagger}$ \\ Departament de Matemàtiques \\ Universitat Autònoma de Barcelona \\ 08193 Bellaterra (Barcelona), Spain \\ quer@mat.uab.cat
}

August 9, 2018

\begin{abstract}
In this note, we establish optimal lower and upper Gaussian bounds for the density of the solution to a class of stochastic integral equations driven by an additive spatially homogeneous Gaussian random field. The proof is based on the techniques of the Malliavin calculus and a density formula obtained by Nourdin and Viens in 14. Then, the main result is applied to the mild solution of a general class of SPDEs driven by a Gaussian noise which is white in time and has a spatially homogeneous correlation. In particular, this covers the case of the stochastic heat and wave equations in $\mathbb{R}^{d}$ with $d \geq 1$ and $d \leq 3$, respectively. The upper and lower Gaussian bounds have the same form and are given in terms of the variance of the stochastic integral term in the mild form of the equation.
\end{abstract}

Key words: Gaussian density estimates, Malliavin calculus, spatially homogeneous Gaussian noise, stochastic partial differential equations

AMS Subject Classification: 60H07, 60H15

* Supported by the NSF grant DMS-0904538.

†Supported by the grant MEC-FEDER Ref. MTM20006-06427 from the Dirección General de Investigación, Ministerio de Educación y Ciencia, Spain. 


\section{Introduction and main result}

Let $X=\left\{X(t, x),(t, x) \in[0, T] \times \mathbb{R}^{d}\right\}$ be a zero mean Gaussian process with an homogeneous covariance function of the form

$$
E(X(t, x) X(s, y))=\Phi(t, s, x-y),
$$

where we assume that $t \mapsto \Phi(t, t, 0)$ is continuous, $\Phi(t, t, 0)>0$ for any $t>0$, and $\Phi(t, 0, x)=0$ for all $(t, x) \in[0, T] \times \mathbb{R}^{d}$.

The purpose of this note is to establish optimal Gaussian lower and upper bounds for the probability density of the solution $u(t, x)$ to the following stochastic integral equation:

$$
u(t, x)=X(t, x)+\int_{0}^{t} \int_{\mathbb{R}^{d}} b(u(s, x-y)) \Gamma(t-s, d y) d s,
$$

where $(t, x) \in[0, T] \times \mathbb{R}^{d}$ and $\Gamma(t, d y)$ is a nonnegative measure such that

$$
\sup _{0 \leq t \leq T} \Gamma\left(t, \mathbb{R}^{d}\right)<+\infty .
$$

Note that if $b: \mathbb{R} \rightarrow \mathbb{R}$ is Lipschitz continuous, there exists a unique solution of (11) and the process $u$ is also homogeneous is the space variable.

In particular, these equations include mild solutions to a large class of SPDEs with additive noise of the form

$$
L u(t, x)=b(u(t, x))+\sigma \dot{W}(t, x), \quad(t, x) \in[0, T] \times \mathbb{R}^{d},
$$

where $L$ denotes a second order differential operator with constant coefficients, the random perturbation $\dot{W}(t, x)$ stands for a Gaussian noise which is white in time and has a spatially homogeneous correlation, and $\sigma$ is constant. We assume here vanishing initial conditions. Let us denote by $\mu$ the spectral measure associated to the noise $\dot{W}$ (for the precise description of the noise, see Section (4). The process $X(t, x)$ is in this case

$$
X(t, x)=\sigma \int_{0}^{t} \int_{\mathbb{R}^{d}} \Gamma(t-s, x-y) W(d s, d y),
$$

where $\Gamma$ denotes the fundamental solution associated to $L$ and, if we simply denote $\Phi(t):=\Phi(t, t, 0)$, then

$$
\Phi(t)=\int_{0}^{t} \int_{\mathbb{R}^{d}}|\mathcal{F} \Gamma(s)(\xi)|^{2} \mu(d \xi) d s,
$$

This has to be a finite quantity for $X(t, x)$ to be well-defined. Let us point out that the main examples of SPDEs of the form (3) to which our results will apply are the stochastic heat equation in any space dimension and the stochastic wave equation in $\mathbb{R}^{d}$ with $d \leq 3$. Indeed, these types of SPDEs have been widely studied during the last two decades, see e.g. 24, 2, 12, 3, 20, 19.

The main result of the paper is the following: 
Theorem 1 Assume that $\Gamma$ satisfies (2) and $b$ is of class $\mathcal{C}^{1}$ with a bounded derivative. Then, there exists $T_{0}>0$ such that for all $(t, x) \in\left(0, T_{0}\right) \times \mathbb{R}^{d}$, the random variable $u(t, x)$ solution to (1) has a density $p$ satisfying, for almost every $z \in \mathbb{R}$ :

$\frac{E|u(t, x)-m|}{C_{2} \Phi(t)} \exp \left\{-\frac{(z-m)^{2}}{C_{1} \Phi(t)}\right\} \leq p(z) \leq \frac{E|u(t, x)-m|}{C_{1} \Phi(t)} \exp \left\{-\frac{(z-m)^{2}}{C_{2} \Phi(t)}\right\}$,

where $m=E(u(t, x))$ and the constants $C_{1}, C_{2}$ are positive and only depend on $b$ and $T_{0}$.

In order to prove Theorem 1 we will apply the techniques of the Malliavin calculus. Indeed, as it has been also done in [18, we will make use of the recent results obtained by Nourdin and Viens in [14. In this paper, the authors provide sufficient conditions on a one-dimensional Wiener functional (that is, on a random variable on the Wiener space) so that its law has a density and it is given by an explicit formula (see [14, Theorem 3.1 and Corollary 3.3]). As it will be made precise in the proof of Theorem 1 (see Section 3), the application of the above-mentioned result of Nourdin and Viens will require a careful analysis of the Malliavin derivative of the solution of equation (1).

We should also remark that recently, there have been an increasing interest in applying the techniques of the Malliavin calculus in order to establish Gaussian lower bounds for the probability density of a general class of Wiener functionals. In particular, this has been applied to diffusion processes and solutions to SPDEs, and the article by Kusuoka and Stroock [9] can be considered as the starting point of this methodology. Therein, a Gaussian type lower bound for the density of a uniformly hypoelliptic diffusion whose drift is a smooth combination of its diffusion coefficient was established. Later on, Kohatsu-Higa [8] got rid of that dependence constraint on the drift and, moreover, took the ideas of Kusuoka and Stroock in order to construct a general method to prove that the density of a multidimensional functional of the Wiener sheet in $[0, T] \times \mathbb{R}^{d}$ admits Gaussian lower bounds (see [7). Then, in the latter paper, KohatsuHiga dealt with a one-dimensional stochastic heat equation in $[0,1]$ (for a related result, see also [18, Theorem 3.1]) and his result was also applied by Dalang and Nualart [4 in the case of a one-dimensional reduced wave equation. The ideas of [7] have also been further developed by Bally [1] to obtain Gaussian lower bounds for locally elliptic Itô processes. Eventually, another recent method for deriving Gaussian lower bounds for multidimensional Wiener functionals has been obtained by Malliavin and Nualart [10] (see [15] for a one-dimensional version of this result). This method is based on an exponential moment condition on the divergence of a covering vector field associated to the Wiener functional.

The paper is organised as follows. In Section 2, we will briefly recall the main tools of the Malliavin calculus needed in the proof of the main result. In particular, we will recall the main points of the method by Nourdin and Viens [14. Section 3 is devoted to prove Theorem 1. Finally, in Section 4, we apply Theorem 1 to the solution of a general spatially homogeneous SPDE. 


\section{Preliminaries}

In this section, we will briefly describe the Gaussian setting in which we will apply the techniques of the Malliavin calculus as well as the method of Nourdin and Viens 14. For a more complete account on these methodologies, we refer the reader to [16] and [14, Sections 2 and 3], respectively.

We denote by $\mathcal{H}$ the Gaussian space generated by the Gaussian process $\left\{X(t, x), \quad(t, x) \in[0, T] \times \mathbb{R}^{d}\right\}$; for a complete account on Gaussian Hilbert spaces, we refer the reader to [6, Chapter 1].

Then, as usual, we denote by $D$ the Malliavin derivative, defined as a closed and unbounded operator from $L^{2}(\Omega)$ into $L^{2}(\Omega ; \mathcal{H})$, whose domain is denoted by $\mathbb{D}^{1,2}$. Thus, for any random variable $F$ belonging to $\mathbb{D}^{1,2}$, its Malliavin derivative $D F$ defines an element in $L^{2}(\Omega ; \mathcal{H})$.

Another important operator in the Malliavin calculus theory that plays an important role in [14] is the generator of the Orstein-Uhlenbeck semigroup (see [16. Section 1.4]). It is usually denoted by $L$ and can be defined by means of its Wiener chaos expansion:

$$
L=\sum_{n=0}^{\infty}-n J_{n}
$$

where $J_{n}$ denotes the projection onto the $n$th Wiener chaos.

In [14], the authors consider a random variable $F \in \mathbb{D}^{1,2}$ with mean zero and define the following function in $\mathbb{R}$ :

$$
g_{F}(z):=E\left[\left\langle D F,-D L^{-1} F\right\rangle_{\mathcal{H}} \mid F=z\right],
$$

where $L^{-1}$ denotes the pseudo-inverse of the generator of the Orstein-Uhlenbeck semigroup $L$. We observe that, by [13, Proposition 3.9], it holds $g(z) \geq 0$ on the support of $F$. Then, Nourdin and Viens prove the following result (see [14, Theorem 3.1 and Corollary 3.3]):

Theorem 2 Assume that there exists a positive constant $c_{1}$ such that

$$
g_{F}(F) \geq c_{1}, \quad \text { a.s. }
$$

Then, the law of $F$ has a density $\rho$ whose support is $\mathbb{R}$ and satisfies, almost everywhere in $\mathbb{R}$ :

$$
\rho(z)=\frac{E|F|}{2 g_{F}(z)} \exp \left(-\int_{0}^{z} \frac{y}{g_{F}(y)} d y\right) .
$$

As stated in [14, Corollary 3.5], an immediate consequence of the above theorem is that, if one also has that $g_{F}(F) \leq c_{2}$, a.s., then the density $\rho$ satisfies, for almost all $z \in \mathbb{R}$ :

$$
\frac{E|F|}{2 c_{1}} \exp \left(-\frac{z^{2}}{2 c_{2}}\right) \leq \rho(z) \leq \frac{E|F|}{2 c_{2}} \exp \left(-\frac{z^{2}}{2 c_{1}}\right) .
$$


In order to deal with particular applications of this method, [14, Proposition 3.7] establishes an alternative formula for $g_{F}(F)$. Indeed, given a random variable $F \in \mathbb{D}^{1,2}$, one can write $D F=\Phi_{F}(W)$, where $\Phi_{F}$ is a measurable mapping from $\mathbb{R}^{\mathcal{H}}$ to $\mathcal{H}$, determined $\left(P \circ W^{-1}\right)$-almost surely (see [16, p. 54-55). Then, it holds that

$$
g_{F}(F)=\int_{0}^{\infty} e^{-\theta} \mathbf{E}\left[\left\langle\Phi_{F}(W), \Phi_{F}\left(e^{-\theta} W+\sqrt{1-e^{-2 \theta}} W^{\prime}\right)\right\rangle_{\mathcal{H}} \mid F\right] d \theta,
$$

where $W^{\prime}$ stands for an independent copy of $W$ such that $W$ and $W^{\prime}$ are defined on the product probability space $\left(\Omega \times \Omega^{\prime}, \mathcal{F} \otimes \mathcal{F}^{\prime}, P \times P^{\prime}\right)$. Eventually, $\mathbf{E}$ denotes the mathematical expectation with respect to $P \times P^{\prime}$.

Let us observe that formula (41) can be still rewritten in the following form:

$$
g_{F}(F)=\int_{0}^{\infty} e^{-\theta} E\left[E^{\prime}\left(\langle D F, \widetilde{D F}\rangle_{\mathcal{H}}\right) \mid F\right] d \theta,
$$

where, for any random variable $X$ defined in $(\Omega, \mathcal{F}, P), \widetilde{X}$ denotes the shifted random variable in $\Omega \times \Omega^{\prime}$

$$
\widetilde{X}\left(\omega, \omega^{\prime}\right)=X\left(e^{-\theta} \omega+\sqrt{1-e^{-2 \theta}} \omega^{\prime}\right), \omega \in \Omega, \omega^{\prime} \in \Omega .
$$

Notice that, indeed, $\widetilde{X}$ depends on the parameter $\theta$, but we have decided to drop its explicit dependence for the sake of simplicity.

\section{Proof of the main result}

This section is devoted to prove Theorem 1, To begin with, we have the following result, whose proof is straightforward and omitted.

Proposition 3 Suppose that $\Gamma$ satisfies condition (2) and $b: \mathbb{R} \rightarrow \mathbb{R}$ is of class $\mathcal{C}^{1}$ with a bounded derivative. Let $u(t, x)$ be the solution to Equation (1). Then $u(t, x)$ belongs to the space $\mathbb{D}^{1,2}$ and

$$
D u(t, x)=X(t, x)+\int_{0}^{t} \int_{\mathbb{R}^{d}} b^{\prime}(u(s, x-y)) D u(s, x-y) \Gamma(t-s, d y) d s,
$$

a.s. for all $(t, x) \in(0, T] \times \mathbb{R}^{d}$.

Let us remark that the pathwise integral in (5) takes values in the Hilbert space $\mathcal{H}$ and can be defined using a standard procedure (see e.g. [17, p. 292]).

Owing to Theorem 2, Theorem 11 will be a consequence of the following proposition. For $(t, x) \in(0, T] \times \mathbb{R}^{d}$ we set $F:=u(t, x)-E(u(t, x))$, so that we need to find almost sure upper and lower bounds for the random variable $g_{F}(F)$ :

$$
g_{F}(F)=\int_{0}^{\infty} e^{-\theta} E\left[E^{\prime}\left(\langle D u(t, x), \widetilde{D u(t, x)}\rangle_{\mathcal{H}}\right) \mid F\right] d \theta .
$$


Proposition 4 Under the same hypothesis as in Theorem [1, there exists $T_{0}>0$ such that, for all $t \in\left[0, T_{0}\right)$ :

$$
C_{1} \Phi(t) \leq g(F) \leq C_{2} \Phi(t), \quad \text { a.s. }
$$

where $C_{1}, C_{2}$ are positive constants depending on $b$ and $T_{0}$.

For the proof of this proposition, we need the following lemma. Recall that $\Phi(t):=\Phi(t, t, 0)=E\left(X(t, x)^{2}\right)$.

Lemma 5 There is a positive constant $C$ such that, for all $t>0$ :

$$
\sup _{\substack{0 \leq r \leq t \\ y \in \mathbb{R}^{d}}} E\left[\|D u(r, y)\|_{\mathcal{H}} \mid F\right] \leq C \sqrt{\Phi(t)}
$$

and

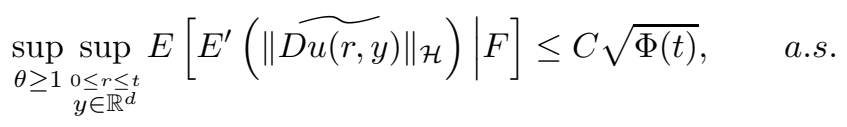

Proof. From (5) and applying Minkowski inequality, we get:

$$
\|D u(t, y)\|_{H} \leq \sqrt{\Phi(t)}+\left\|b^{\prime}\right\|_{\infty} \int_{0}^{t} \int_{\mathbb{R}^{d}}\|D u(s, y-z)\|_{H} \Gamma(t-s, d z) d s .
$$

As a consequence, we have the following estimate:

$$
\begin{aligned}
E\left[\|D u(t, y)\|_{H} \mid F\right] \leq & \sqrt{\Phi(t)}+\left\|b^{\prime}\right\|_{\infty} \\
& \times \int_{0}^{t} \int_{\mathbb{R}^{d}} E\left[\|D u(s, y-z)\|_{H} \mid F\right] \Gamma(t-s, d z) d s .
\end{aligned}
$$

Set

$$
Y_{t}:=\sup _{\substack{0 \leq r \leq t \\ y \in \mathbb{R}^{d}}} E\left[\|D u(r, y)\|_{H} \mid F\right] .
$$

Then, we have proved that

$$
Y_{t} \leq \sqrt{\Phi(t)}+\left\|b^{\prime}\right\|_{\infty} \int_{0}^{t} Y_{s} \Gamma\left(t-s, \mathbb{R}^{d}\right) d s,
$$

and a suitable generalisation of Gronwall lemma (e.g. [3, Lemma 15]) allows us to conclude the proof.

Proof of Proposition 4. It follows the same lines as in the proofs of Propositions 4.5 and 5.5 in [18. Indeed, one just needs to be careful and try to keep the sharpest bounds which appear throughout the proof. Let us be more precise about this method, namely, by (5) and (6), we have that

$$
g_{F}(F)=\Phi(t)+\sum_{i=1}^{3} A_{i}(t, x)
$$


where

$$
\begin{aligned}
& A_{1}(t, x)=E\left[\int_{0}^{t} \int_{\mathbb{R}^{d}} b^{\prime}(u(s, x-y))\langle X(t, x), D u(s, x-y)\rangle_{\mathcal{H}} \Gamma(t-s, d y) d s \mid F\right] \\
& A_{2}(t, x)=\int_{0}^{\infty} e^{-\theta} E\left[E ^ { \prime } \left(\int_{0}^{t} \int_{\mathbb{R}^{d}} b^{\prime}(u(\widetilde{s, x-y}))\right.\right. \\
& \left.\left.\times\langle X(t, x), D u \widetilde{(s, x-y)}\rangle_{\mathcal{H}} \Gamma(t-s, d y) d s\right) \mid F\right] d \theta, \\
& A_{3}(t, x)=\int_{0}^{\infty} e^{-\theta} E\left[E ^ { \prime } \left(\int_{[0, t]^{2}} \int_{\mathbb{R}^{2 d}} b^{\prime}(u(s, x-y)) b^{\prime}(\widetilde{(u+x-z)})\right.\right. \\
& \left.\left.\times\langle D u(s, x-y), D \widetilde{(r, x-z)}\rangle_{\mathcal{H}} \Gamma(t-s, d y) \Gamma(t-r, d z) d r d s\right) \mid F\right] d \theta .
\end{aligned}
$$

Let us first prove the lower bound. For this, observe that we have

$$
g_{F}(F) \geq \Phi(t)-\left|A_{1}(t, x)+A_{2}(t, x)+A_{3}(t, x)\right| .
$$

Let us bound $\left|A_{1}(t, x)\right|$. We will use the following notation:

$$
\Psi(t):=\int_{0}^{t} \Gamma\left(s, \mathbb{R}^{d}\right) d s .
$$

Notice that, by hypothesis, $\sup _{t \in[0, T]} \Psi(t)<+\infty$ and in fact $\Psi(t)$ converges to 0 as $t$ tends to 0 . We can write, applying (7) in Lemma 5 and using the notation (9):

$$
\begin{aligned}
\left|A_{1}(t, x)\right| & \leq \sqrt{\Phi(t)}\left\|b^{\prime}\right\|_{\infty} \mathbb{E}\left[\int_{0}^{t} \int_{\mathbb{R}^{d}}\|D u(s, x-y)\|_{\mathcal{H}} \Gamma(t-s, d y) d s \mid F\right] \\
& \leq \sqrt{\Phi(t)}\left\|b^{\prime}\right\|_{\infty}\left(\int_{0}^{t} \Gamma\left(s, \mathbb{R}^{d}\right) d s\right) \sup _{\substack{0 \leq s \leq t \\
z \in \mathbb{R}^{d}}} \mathbb{E}\left[\|D u(s, z)\|_{\mathcal{H}} \mid F\right] \\
& \leq C \Phi(t)\left\|b^{\prime}\right\|_{\infty}\left(\int_{0}^{t} \Gamma\left(s, \mathbb{R}^{d}\right) d s\right) \\
& =C\left\|b^{\prime}\right\|_{\infty} \Phi(t) \Psi(t) .
\end{aligned}
$$

For the analysis of the term $\left|A_{2}(t, x)\right|$, one can proceed using exactly the same arguments (the expectation $E^{\prime}$ and the integral with respect to $\theta$ do not affect the final result), but here we will need to apply (8) in Lemma 5. Indeed, one also obtains that

$$
\left|A_{2}(t, x)\right| \leq C\left\|b^{\prime}\right\|_{\infty} \Phi(t) \Psi(t) .
$$

Eventually, in order to bound $\left|A_{3}(t, x)\right|$, we observe that

$$
\begin{aligned}
\left|A_{3}(t, x)\right| \leq \| & b^{\prime} \|_{\infty}^{2} \int_{0}^{\infty} e^{-\theta} \int_{[0, t]^{2}} \int_{\mathbb{R}^{2 d}} \\
& \times E\left[\|D u(s, x-y)\|_{\mathcal{H}} E^{\prime}\left(\| D u\left(\widetilde{(r, x-z)} \|_{\mathcal{H}}\right) \mid F\right]\right. \\
& \times \Gamma(t-s, d y) \Gamma(t-r, d z) d r d s d \theta .
\end{aligned}
$$


for which we apply Cauchy-Schwartz inequality with respect to the conditional expectation with respect to $F$ and we use the estimate

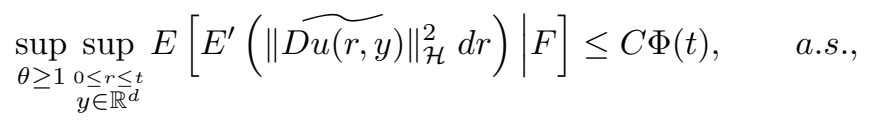

whose proof is similar to that of (8). In this way, we obtain that

$$
\left|A_{3}(t, x)\right| \leq C\left\|b^{\prime}\right\|_{\infty}^{2} \Phi(t) \Psi(t)^{2} .
$$

Putting together the bounds for $\left|A_{i}(t, x)\right|, i=1,2,3$, we have that there are positive constants $k_{1}$ and $k_{2}$ (only depending on $b$ ) such that, for all $t \in(0, T]$ and $x \in \mathbb{R}^{d}$ (recall that $F=u(t, x)-E(u(t, x))$ ),

$$
\begin{aligned}
g_{F}(F) & \geq k_{1} \Phi(t)-k_{2}\left(\Phi(t) \Psi(t)+\Phi(t) \Psi(t)^{2}\right) \\
& =\Phi(t)\left(k_{1}-k_{2}\left(\Psi(t)+\Psi(t)^{2}\right)\right),
\end{aligned}
$$

where we remind that $\Psi(t)=\int_{0}^{t} \Gamma\left(s, \mathbb{R}^{d}\right) d s$. Hence, if $t$ is sufficiently small, say $t<T_{0}$ with $T_{0}>0$ satisfying $k_{1}-k_{2}\left(\Psi\left(T_{0}\right)+\Psi\left(T_{0}\right)^{2}\right)>0$, then

$$
g(F) \geq C_{1} \Phi(t),
$$

where $C_{1}$ is a positive constant depending on $b$ and $T_{0}$. This proves the lower bound in the statement.

Concerning the upper bound, observe that we have, for all $t \in\left(0, T_{0}\right)$ :

$$
\begin{aligned}
g_{F}(F) & \leq \Phi(t)+\sum_{i=1}^{3}\left|A_{i}(t, x)\right| \\
& \leq C \Phi(t)\left(1+\Psi\left(T_{0}\right)+\Psi\left(T_{0}\right)^{2}\right) \\
& \leq C_{2} \Phi(t),
\end{aligned}
$$

where $C_{2}$ is a positive constant depending on $b$ and $T_{0}$. This concludes the proof.

\section{Application to spatially homogeneous SPDEs}

As we commented in the Introduction, the main examples of equations of the form (1) which Theorem 1 can be applied to correspond to the mild formulation of the following SPDEs:

$$
L u(t, x)=b(u(t, x))+\sigma \dot{W}(t, x), \quad(t, x) \in[0, T] \times \mathbb{R}^{d},
$$

where $L$ is a second order differential operator with constant coefficients and $\dot{W}$ is a Gaussian noise which is white in time and has some spatially homogeneous correlation. More precisely, on some probability space $(\Omega, \mathcal{F}, P)$, we consider a family of centered Gaussian random variables $W=\left\{W(\varphi), \varphi \in \mathcal{C}_{0}^{\infty}\left(\mathbb{R}^{d+1}\right)\right\}$, 
where $\mathcal{C}_{0}^{\infty}\left(\mathbb{R}^{d+1}\right)$ denotes the space of infinitely differentiable functions with compact support, with the following covariance functional:

$$
E(W(\varphi) W(\psi))=\int_{0}^{\infty} \int_{\mathbb{R}^{d}}\left(\varphi(t) * \psi_{(s)}(t)\right)(x) \Lambda(d x) d t, \quad \varphi, \psi \in \mathcal{C}_{0}^{\infty}\left(\mathbb{R}^{d+1}\right),
$$

where $\psi_{(s)}(t, x):=\psi(t,-x)$ and $\Lambda$ is a non-negative and non-negative definite tempered measure. For the right-hand side of (11) to define a covariance functional, it turns out that $\Lambda$ has to be symmetric and the Fourier transform of a non-negative tempered measure $\mu$ (see [23, Chapter VII, Théorème XVII]). Usually, $\mu$ is called the spectral measure of the noise $W$. Let us denote by $\left(\mathcal{F}_{t}\right)_{t}$ the filtration generated by $W$, conveniently completed.

Usual examples of spatial correlations are given by measures of the form $\Lambda(d x)=f(x) d x$, where $f$ is a non-negative and continuous function on $\mathbb{R}^{d} \backslash\{0\}$, which is integrable in a neighbourhood of 0 . For instance, one can consider a Riesz kernel $f(x)=|x|^{-\epsilon}$, for $0<\epsilon<d$, while the space-time white noise corresponds to consider $f=\delta_{0}$; in this latter case, the spectral measure is the Lebesgue measure on $\mathbb{R}^{d}$.

By definition, a mild solution of (10) is a $\mathcal{F}_{t}$-adapted stochastic process $\left\{u(t, x),(t, x) \in[0, T] \times \mathbb{R}^{d}\right\}$ satisfying

$$
\begin{aligned}
u(t, x)=\sigma \int_{0}^{t} & \int_{\mathbb{R}^{d}} \Gamma(t-s, x-y) W(d s, d y) \\
& +\int_{0}^{t} \int_{\mathbb{R}^{d}} b(u(s, x-y)) \Gamma(t-s, d y) d s,
\end{aligned}
$$

a.s. for all $(t, x) \in[0, T] \times \mathbb{R}^{d}$, where $\Gamma$ denotes the fundamental solution associated to $L$. Without any loose of generality, we can assume that $\sigma=1$. Let us point out that the stochastic integral in the right-hand side of equation (12) takes values in $\mathbb{R}$ and is considered as an integral with respect to the cylindrical Wiener process associated to $W$, as described in [17, Section 3]. Note, however, that this stochastic integral can be also defined in the sense of Dalang [3] (see also [24]) or even in the more abstract framework of Da Prato and Zabczyk [5].

In this setting, the process $X$ is given by

$$
X(t, x)=\int_{0}^{t} \int_{\mathbb{R}^{d}} \Gamma(t-s, x-y) W(d s, d y),
$$

for which one verifies that

$$
E(X(t, x) X(s, y))=\int_{0}^{s \wedge t} \int_{\mathbb{R}^{d}} e^{2 \pi i(x-y)} \mathcal{F} \Gamma(t-r)(\xi) \overline{\mathcal{F} \Gamma(s-r)(\xi)} \mu(d \xi) d r .
$$

Hence, in particular,

$$
\Phi(t)=E\left(X(t, x)^{2}\right)=\int_{0}^{t} \int_{\mathbb{R}^{d}}|\mathcal{F} \Gamma(r)(\xi)|^{2} \mu(d \xi) d r, \quad t \in[0, T] .
$$

At this point, we observe that $X(t, x)$ is a well-defined Gaussian random variable whenever the following condition is satisfied (see e.g. [17, Lemma 3.2]): 
Hypothesis 6 For all $t>0, \Gamma(t)$ defines a non-negative distribution with rapid decrease such that

$$
\int_{0}^{T} \int_{\mathbb{R}^{d}}|\mathcal{F} \Gamma(t)(\xi)|^{2} \mu(d \xi) d t<\infty .
$$

Moreover, $\Gamma$ is a non-negative measure of the form $\Gamma(t, d x) d t$ such that, for all $T>0$,

$$
\sup _{0 \leq t \leq T} \Gamma\left(t, \mathbb{R}^{d}\right) \leq C_{T}<\infty .
$$

Thus, Theorem 1 applied to equation (12) reads:

Theorem 7 Assume that Hypothesis [6 is satisfied and $b$ is of class $\mathcal{C}^{1}$ with a bounded derivative. Then, there exists $T_{0}>0$ such that for all $(t, x) \in\left(0, T_{0}\right) \times$ $\mathbb{R}^{d}$, the random variable $u(t, x)$ solution to (12) has a density $p$ satisfying, for almost every $z \in \mathbb{R}$ :

$\frac{E|u(t, x)-m|}{C_{2} \Phi(t)} \exp \left\{-\frac{(z-m)^{2}}{C_{1} \Phi(t)}\right\} \leq p(z) \leq \frac{E|u(t, x)-m|}{C_{1} \Phi(t)} \exp \left\{-\frac{(z-m)^{2}}{C_{2} \Phi(t)}\right\}$,

where $m=E(u(t, x))$ and the constants $C_{1}, C_{2}$ are positive and only depend on $b$ and $T_{0}$.

Example 8 Theorem 7 applies to the case of the stochastic heat equation in any space dimension and the stochastic wave equation in $\mathbb{R}^{d}$ with $d \leq 3$. Indeed, in both examples, Hypothesis [6 is fulfilled if and only if

$$
\int_{\mathbb{R}^{d}} \frac{1}{1+|\xi|^{2}} \mu(d \xi)<+\infty
$$

(see e.g. [3, Section 3]). As a consequence, in the case of the stochastic wave equation, Theorem 7 exhibits an improvement of [18, Theorem 5.3], where the lower and upper Gaussian bounds were not optimal and a slightly stronger condition on $\mu$ was assumed.

Remark 9 Independently of the results in [14], the study of the existence and smoothness of the density for SPDEs of the form (10) (in particular for stochastic heat and wave equations), has already been tackled by several authors. Namely, let us mention the works [2, 12, 11, 21, 22, 17].

\section{References}

[1] Bally, V. Lower bounds for the density of locally elliptic Itô processes. Ann. Probab. 34 (2006), no. 6, 2406-2440.

[2] Carmona, R.; Nualart, D. Random nonlinear wave equations: Smoothness of the solutions. Probab. Theory Relat. Fields 79, No.4, 469-508 (1988). 
[3] Dalang, R.C. Extending martingale measure stochastic integral with applications to spatially homogeneous s. p. d. e's. Electron. J. Probab. 4 (1999), no. 6,29 pp.

[4] Dalang, R.C.; Nualart, E. Potential theory for hyperbolic SPDEs. Ann. Probab. 32 (2004), no. 3A, 2099-2148.

[5] Da Prato, G.; Zabczyk, J. Stochastic equations in infinite dimensions. Cambridge University Press, Cambridge, 1992.

[6] Janson, S. Gaussian Hilbert spaces. Cambridge Tracts in Mathematics, 129. Cambridge University Press, Cambridge, 1997.

[7] Kohatsu-Higa, A. Lower bounds for densities of uniformly elliptic random variables on Wiener space. Probab. Theory Related Fields 126 (2003), no. 3, 421-457.

[8] Kohatsu-Higa, A. Lower bounds for densities of uniformly elliptic nonhomogeneous diffusions, Proceedings of the Stochastic Inequalities Conference in Barcelona, Progr. Probab. vol. 56 (2003), pp. 323-338.

[9] Kusuoka, D.; Stroock, D. Applications of the Malliavin calculus, part III. J. Fac. Sci. Univ. Tokyo Sect. IA Math. 34 (1987), 391-442.

[10] Malliavin, P.; Nualart, E. Density minoration of a strongly non degenerated random variable. J. Funct. Anal. 256 (2009), no. 12, 4197-4214.

[11] Márquez-Carreras, D.; Mellouk, M.; Sarrà, M. On stochastic partial differential equations with spatially correlated noise: smoothness of the law. Stoch. Proc. Appl. 93 (2001), 269-284.

[12] Millet, A.; Sanz-Solé, M. A stochastic wave equation in two space dimensions: smoothness of the law. Ann. Probab. 27, No.2, 803-844 (1999).

[13] Nourdin, I.; Peccati, G. Stein's method on Wiener chaos. Probab. Theory and Rel. Fields 145, No. 1-2, 75-118 (2009).

[14] Nourdin, I.; Viens, F. Density estimates and concentration inequalities with Malliavin calculus. Electronic Journal of Probability 14, Paper No. 78, 2287-2309 (2009).

[15] Nualart, E. Exponential divergence estimates and heat kernel tail. C. R. Math. Acad. Sci. Paris 338 (2004), no. 1, 77-80.

[16] Nualart, D. The Malliavin Calculus and Related Topics, Second edition. Springer-Verlag, Berlin (2006).

[17] Nualart, D.; Quer-Sardanyons, L. Existence and smoothness of the density for spatially homogeneous SPDEs. Potential Anal. 27 (2007), no. 3, 281299. 
[18] Nualart, D.; Quer-Sardanyons, L. Gaussian density estimates for solutions to quasi-linear stochastic partial differential equations. Stoch. Processes and their Appl. 119, 3914-3938 (2009).

[19] Peszat, S. The Cauchy problem for a nonlinear stochastic wave equation in any dimension. J. Evol. Equ. 2 (2002), no. 3, 383-394.

[20] Peszat, S.; Zabczyk, J. Stochastic evolution equations with a spatially homogeneous Wiener process. Stochastic Process. Appl. 72 (1997), no. 2, 187-204.

[21] Quer-Sardanyons, L.; Sanz-Solé, M. Absolute continuity of the law of the solution to the 3-dimensional stochastic wave equation. J. Funct. Anal. 206 (2004), no.1, 1-32.

[22] Quer-Sardanyons, L.; Sanz-Solé, M. A stochastic wave equation in dimension 3: smoothness of the law. Bernoulli 10 (2004), no. 1, 165-186.

[23] Schwartz, L. Théorie des distributions. Hermann, Paris (1966).

[24] Walsh, J. B. An introduction to stochastic partial differential equations. In: Hennequin, P. L. (ed.) École d'été de probabilités de Saint-Flour XIV - 1984, Lect. Notes Math. vol. 1180, pp. 265-437. Springer, Berlin (1986). 